\title{
Effect of different training models on motoric and swimming performance in prepubescent swimmers
}

\author{
İsmet Alagöz ${ }^{1 \mathrm{ABCD}}$, Sema Can ${ }^{2 \mathrm{AD}}$, Erkan Demirkan ${ }^{2 \mathrm{AD}}$, Tuğrul Özkadi ${ }^{3 \mathrm{AB}}$, Emre Demir $^{4 \mathrm{CD}}$ \\ ${ }^{1}$ Ministry of Youth and Sports, Turkey \\ ${ }^{2}$ Faculty of Sport Sciences, Department of Coaching Training, Hitit University, Turkey \\ ${ }^{3}$ Department of Sport Management, Hitit University, Turkey \\ ${ }^{4}$ Faculty of Medicine, Department of Biostatistics, Hitit University, Turkey
}

Authors' Contribution: A - Study design; B - Data collection; C - Statistical analysis; D - Manuscript Preparation; E - Funds Collection

\begin{abstract}
Background The aim of this study was to examine the effects of different training programs on the improvement of and Study Aim motoric and swimming performance prepubescent swimmers.

Material and Forty-five children between the ages of 9-11 years with at least 2 years of training experiences, participated Methods in the study. Three different [(1) dry-land with elastic resistance band group + swimming (ERB); (2) dry-land without elastic resistance band (DL) + swimming and (3) swimming group (SG) with swimming training alone] training group were formed. And a 12-week training program was implemented thought the study. Biceps, chest, waist, hip, thigh body circumference measurements were taken from all participants. Vertical jump (VJ), flexed-arm strength (FAS), speed, upper body strength (UBS), Standing horizontal jump $(\mathrm{SHJ})$, flexibility, aerobic endurance (AE), balance, and $50 \mathrm{~m}$ freestyle swimming (FS) score were tested on the participants. As statistical analysis, normality and homogeneity of variance assumption were checked (Shapiro-Wilk and Levene tests, respectively). A non-normal distribution was found. The values of each variable were expressed as mean \pm standard deviation, and median. The training effects within the groups were evaluated using analyses of Friedman for repeated measures and the level of significance was set at $\mathrm{p}<0.05$ for all tests.
\end{abstract}

Results There was a significant difference in SHJ, UBS, FAS, speed, and FS score among the assessment times 1-3 and $1-4$ in both of ERB and DL training groups $(p<0.05)$. ERB and DL training were significantly effective compared to the SG on VJ, FAS, speed, UBS, and freestyle swimming performance $(p<0.05)$.

Conclusions: The study findings showed that DL training more effected relatively on motoric performance.

Keywords: $\quad$ children, athletes, freestyle, dry-land training, elastic band.

\section{Introduction}

In most sports, strength and conditioning training regimes are required to enhance performance and prevent injuries. Swimming is a complex sport that requires multifactorial training involving endurance, strength, power, speed, agility, and anthropometrics. Many studies reported that the effectiveness of these components on swimming performance was clear [1-4].

Scientists and coaches report that swimming training should consist of both dry-land and in-water sessions. Therefore, the researchers focused on not only in-water training, but also on dry-land training, evincing its contribution to a better swimming performance [3, 5-7]. In order to enhance the physical condition, resistance training is an important component of the training program. Following resistance training increase arm strength lead to in higher maximum stroke force, and also improved sprint swim performance [8-10].

However, some resistance training models, including using free weights or resistance-training machines which may have certain risks such as injury, so they may not be suitable particularly for childhood. American Academy

\footnotetext{
○ İsmet Alagöz, Sema Can, Erkan Demirkan, Tuğrul Özkadı, Emre Demir, 2021

doi:10.15561/26649837.2021.0503
}

of Pediatrics has supported to avoid resistance training such as powerlifting, bodybuilding, and maximal lifts for preadolescents and adolescents until reach physical and skeletal maturity [11]. In addition to this, every kind of resistance training may not be similar effect on the conditioning components, including strength, power etc. A study from Cuenca-Fernández et al [12] reported that semi-tethered loaded swimming training showed to be sensitive on improving swimmer's technique rather than increasing physical conditioning. Some studies reported that resistance training would not be the main reason for in itself; either the load was not suitable or the lifting technique caused negative results $[11,13]$. Therefore, the diversities in resistance training regimes may provide both the age requirements and multi-functional improvement for young athletes. Elastic resistance band training, a technique commonly used in rehabilitation, provides a safe and effective progressive overload technique, applicable for people of all ages [14].

Besides, elastic resistance band is types of equipment that are relatively inexpensive, easy to use, portable and safe, thus widely used. It provides advantages with respect to training with free weights or weight machine in rehabilitative medicine [15]. Several studies have investigated the effects of elastic band training on 
performance and healthy life on different groups including athlete, [16-18] patients [19], postmenopausal women, and the elderly [20]. The studies in the literature, there was a lack of research about the effects of ERB training on swimming performance in prepubescent swimmers.

Although there were many resistance training methods implemented, there is a limited knowledge as to whichever methods are better to enhance the multifunctional performance improvements that affected swimming performance. For this reason, it would be of a great worth for the coaches to have a resource of more beneficial resistance training protocols. It may be presented to the information on how it influences swimming performance on prepubescent swimmers. In this study, dry-land training consisted of two different practices including (a) resistance training with self-body weight and medicine ball and (b) only elastic resistance band. Therefore, the main purpose of the current study was (i) to compare the effects of twelve weeks of the dry-land training and in-water training, (ii) to assess the efficacy of resistance training with using self-body weight and medicine ball (DL training) and the elastic resistance band (ERB training) for improvement motoric and swimming performance in prepubescent swimmers.

\section{Materials and Methods}

\section{Participants}

Forty-five prepubescent swimmers (boys: $\mathrm{n}=23$; girls: $\mathrm{n}=22$, the range of age 9-11 year), who have at least 2-year sport experience and free from injuries or health problems, participated in this study. Before of the study, to determine the homogeneity for all groups, physical performances test and circumferences measurements were performed for all participants. Then according to statically analyzes the results there was no found significant differences in variables, including VJ, FAS, speed, UBS, FS score, SHJ, flexibility, endurance, balance, biceps, chest, waist, hip, thigh. Power analyzes test was applied to determine the sample size using for each of the variables. As based on the power analyzed results, it was determined the group size using highest sample size taken from the power analyzed. In addition, the participants did not engage to another sports, engaged to just swimming. The participants were randomly divided into the three groups: elastic resistance band group (boys: $n=8$; girls: $n=7$ ), dryland resistance training group without elastic band (boys: $\mathrm{n}=6$; girls: $\mathrm{n}=9$ ), and swimming group (boys: $\mathrm{n}=9$; girls $\mathrm{n}=6$ ). Tanner stage test was performed by the physician to determine whether the participants entered adolescence or not. As a criterion for the tanner stage, it was taken breast development for female and, external genitalia for male [21]. As a result of the stage test, it was determined that there were no participants who entered the adolescent stage. The written informed consent form was signed by the parents, because the participants were under 18 years of age. The study was conducted by the ethics committee of Hitit University, with the Declaration of Helsinki for research involving human participants (Decision no: 2018-12).

\section{Design and Procedures}

In this study, ERB and DL groups were named as the experimental groups; the control group was named as the swimming group. All measurements were carried out for all variables in each of measurement time by the expert practitioner. The assessments were carried out four times during the study: at the beginning of the study (1), and after four (2), eight (3) and twelve-week of the training session (4). The start of the study, the implemented equipment and procedures were familiarized to the participants. The measurements consisted of the anthropometric and performance tests, including motoric components and swimming performance performed in each of the four evaluation times. During the study, the participants were not involved in any other training program. The participants were instructed not to exercise for at least 24 hours and not to eat for at least three hours before the performance analysis. The performance analysis followed systematic order without affecting results and started with a 10-min warm-up performed by all subjects. The measurements performance analyze were designed according to the criterias including used energy systems, muscle groups, and recovery duration.

\section{Training protocol}

As remarked in the section of the participants, the study groups were separated as the elastic resistance band group, the dry-land resistance training group without the elastic band, and the swimming group. Different venues were used to the training for each group. The ERB and DL training sessions ( 3 sessions per week of 30 minutes each), before in-water training took part in addition to regular swimming training sessions. The swimming training program with 60 minutes was performed as the common training program for experimental groups. Each training session lasted 90 minutes (DL and ERB training: 30 minutes, and the swimming training: 60 minutes; the swimming training lasted 90 minute for the SG). Different color bands, including yellow, red, green, blue, were used in ERB training based on the resistance intensity from mild to hard respectively. In the $3^{\text {rd }}$ set, when 15 repetitions were reached, an upper colored band was used in the next training session. The components such as; the same body parts, set, repeat, and training volume in the selection of exercise- according to the training protocols to provide the equivalent between trainings- were taken into account [22]. The detailed information about the DL and ERB training protocols were presented in Table 1.

Assessment

Test procedures were explained and demonstrated to the participants before each of the tests. All the tests were performed at the same time of the day to avoid any effect of circadian rhythms. Experimental groups and the SG were evaluated at the same moment in the procedures schedule in preliminary, after 4, 8, 12 weeks. In measurements of VJ, SHJ, UBS, FAS, balance, it was given 2-min rest interval between each of the 3 trial and recorded the best score. In measurement of speed, 3-min rest interval was given between each of the 3 trial, and the best scores were recorded. In measurement of flexibility, 
30 second rest interval was given between each of the 3 trial, the best score was recorded to analysis. The subject waited at least 2 seconds at maximum reached distance. In Swimming performance, all subjects completed three $50 \mathrm{~m}$ free style swimming trials with a $15 \mathrm{~min}$ rest period between the trials. The performance analyses were completed including: which included SHJ, and VJ tests to measure explosive power, the UBS test was measured with throwing medicine ball, the FAS test was used to measure static arm strength endurance, the sit and reach test (S\&R) was used to measure hamstrings and lumbar spine flexibility; the cooper test (covered distance during 9 minute) was to measure AE, $30 \mathrm{~m}$ speed test was used to measure acceleration and speed, the flamingo test (count the number of falls in one minute of balancing) was used to measure the ability to balance successfully on a leg, were completed [23].

Anthropometric data included five body circumferences such as biceps flexion, chest, waist, hip, thigh. Besides, the weight was determined within $0.1 \mathrm{~kg}$ for each subject using an electronic scale calibrated before each measurement session (Seca 664, Hamburg, Germany). The height was determined using a fixed wall-scale measuring device to the nearest $0.1 \mathrm{~cm}$. The measurements were obtained using a Holtain anthropometric set by the expert practitioner according to the techniques recommended by Miller [23]. Skinfold thickness ( $\mathrm{mm}$ ) was measured at three identified anatomical landmark sites, including the chest, the abdomen, and the thighs for boys and the triceps, the suprailium, and the thighs for girls, using a Holtain caliper. The Body fat (BF) percentage for boys and girls was calculated by using Jackson-Pollock equation [24].

Statistical Analyses

Normality and homogeneity of variance assumption were checked (Shapiro-Wilk and Levene tests, respectively), and a non-normal distribution was found. Non-parametric tests were conducted. The values of each variable were expressed as mean \pm standard deviation, and median. The training effects within the groups were evaluated using analyses of Friedman for repeated measures. Following the Friedman test, Post hoc analysis with Wilcoxon signed-rank tests was conducted with a Bonferroni correction applied in order to find out which groups (time point) caused the differences. All the analyses were performed with SPSS (version 22.0; SPSS Inc, Chicago, IL), and the level of significance was set at $\mathrm{p}<0.05$ for all tests.

\section{Results}

A total of 45 prepubescent swimmers (15 in ERB, 15 in DL, and 15 in C) were measured at the baseline. At the baseline, there were no significant differences between any of the physical characteristic variables (Table 2), thus, it was provided acceptable homogeneity among the groups.

Table 1. The training programs, according to the groups.

\begin{tabular}{|c|c|c|c|c|c|}
\hline \multicolumn{3}{|c|}{ ERB training protocol } & \multicolumn{3}{|c|}{ Dry-land training protocol } \\
\hline Wednesday & & & Wednesday & & \\
\hline Sunday & Friday & Sets $\mathrm{x}$ reps & Sunday & Friday & Sets $\mathrm{x}$ reps \\
\hline Shoulder flex & Lateral raise & Week 1 - 4 & Ball Slams & Front raise $\mathrm{MB}$ & Week 1 - 4 \\
\hline Knee ext & Minisquat & $3 \times 10$ & Jumping & Squat & $3 \times 8$ \\
\hline Elbow flex & Elbow kick back & Week 5 - 8 & Arm flex MB & Dibs & Week 5 - 8 \\
\hline Back ext & Crunch & $3 \times 10$ & Sit-up & V ups & $3 \times 10$ \\
\hline Elbow ext & Arm curl & Week 9 - 12 & Arm ext MB & Push up & Week $9-12$ \\
\hline Shoulder abd & Front raise & $3 \times 10$ & V push up & V push up & $3 \times 12$ \\
\hline
\end{tabular}

Abbreviations: Abd: Abduction; Add: Adduction; Ext: Extension; Flex: Flexion; MB: Medicine ball (1 kg).

Table 2. The subject characteristics according to the groups.

\begin{tabular}{|c|c|c|c|c|c|c|}
\hline \multirow{2}{*}{ Variables } & \multicolumn{2}{|l|}{ ERB } & \multicolumn{2}{|l|}{ Dry-land } & \multicolumn{2}{|l|}{ Swimming } \\
\hline & Girls & Boys & Girls & Boys & Girls & Boys \\
\hline Age (year) & $10.7 \pm 0.4$ & $9.8 \pm 0.6$ & $9.6 \pm 0.5$ & $9.6 \pm 0.8$ & $10.1 \pm 0.9$ & $10.2 \pm 0.9$ \\
\hline Height (cm) (1) & $146.86 \pm 7.8$ & $144.75 \pm 5.65$ & $140.22 \pm 9.39$ & $142.33 \pm 7.52 /$ & $142.67 \pm 11.86$ & $146.22 \pm 8.88$ \\
\hline Height (cm) (4) & $151.7 \pm 2.93$ & $147.68 \pm 5.59$ & $143.84 \pm 7.53^{*}$ & $145.13 \pm 8.35^{*}$ & $145.23 \pm 10.47^{*}$ & $148.05 \pm 7.68^{*}$ \\
\hline Body mass (kg) (1) & $45.5 \pm 10.06$ & $40 \pm 8.28$ & $38.3 \pm 9.51$ & $35.8 \pm 5.63$ & $38 \pm 10.48$ & $36.1 \pm 5.57$ \\
\hline Body mass (kg) (4) & $47.08 \pm 8.37^{*}$ & $41.21 \pm 6.40 *$ & $39.26 \pm 8.43^{*}$ & $38.38 \pm 5.05^{*}$ & $38.40 \pm 9.25 *$ & $37.98 \pm 3.87^{*}$ \\
\hline Fat (\%) (1) & $12.15 \pm 5.28$ & $11.10 \pm 6.86$ & $12.58 \pm 5.23$ & $11.28 \pm 5.9$ & $13.15 \pm 6.08$ & $8.57 \pm 5.08$ \\
\hline Fat (\%) (4) & $11.99 \pm 5.19$ & $10.99 \pm 6.4$ & $12.6 \pm 5.03$ & $11.11 \pm 5.23$ & $13.05 \pm 5.95$ & $8.55 \pm 5.08$ \\
\hline FFM (kg) (1) & $36.3 \pm 5.78$ & $38.13 \pm 6.6$ & $34.14 \pm 7.18$ & $30.41 \pm 4.70$ & $29.48 \pm 6.31$ & $34.47 \pm 5.90$ \\
\hline FFM (kg) (4) & $38.22 \pm 5.06$ & $38.91 \pm 5.27$ & $35.97 \pm 5.88$ & $31.90 \pm 3.00$ & $30.55 \pm 4.67^{*}$ & $36.78 \pm 4.29 *$ \\
\hline
\end{tabular}

*p<0.05; FFM: Free fat mass; *Pre-study measurement (1); *Post-study measurement (2). 
Figure 1 presents the changes of biceps, chest, waist, hip and thigh circumference values for all groups that were obtained through the study. The results showed that there was a statistically significant difference in biceps and chest circumference between the assessment times 1-3 and 1-4 for the ERB training group. Besides, a statistically significant difference was found in biceps circumference between 1-3 and 1-4 for DL training group. No differences were found for the other parameters.

Figure 2 presents the change of results in-group throughout the study in VJ, FAS, UBS, speed, SHJ, flexibility, balance, AE, and FS score performance components for all groups. For the ERB and DL training groups, there was a statistically significant difference in SHJ, UBS, FAS, speed, and FS score among the assessment times 1-3 and 1-4. For the SG, a statistically significant difference was found in UBS, FAS, speed, FS score, SHJ between the assessment times 1 and 4 . No statistically significant difference was found in balance, endurance and flexibility parameters of any assessment times during the study for all groups.
Table 3 presents both the improvement of performance variables depending on each of the assessment time from the beginning of the training period (1) to the twelveweeks of the training session (4) and the total change difference (TCD) as \% among all groups. There was a significant difference in VJ performance in both the second and third measurement times between the DL and SG $(p<0.001)$. In the fourth measurement, there was a significant difference in VJ performance in both ERB and DL versus $S G(p<0.001)$. In TCD, there was no significant change between the ERB and DL training group, but the change difference was significant in ERB and DL training group comparing to the $\mathrm{SG}(\mathrm{p}<0.05)$. In FAS performance change, there were significant differences in the second, third and fourth measurements, in both ERB and DL versus $\mathrm{SG}(\mathrm{p}<0.001)$. In $\mathrm{TCD}$, there was no significant change between the ERB and DL training group, but the change difference was significantly in ERB and DL training group comparing to the $\mathrm{SG}(\mathrm{p}<0.05)$. In speed performance change, there was a significant difference in both the third and fourth measurement times between the

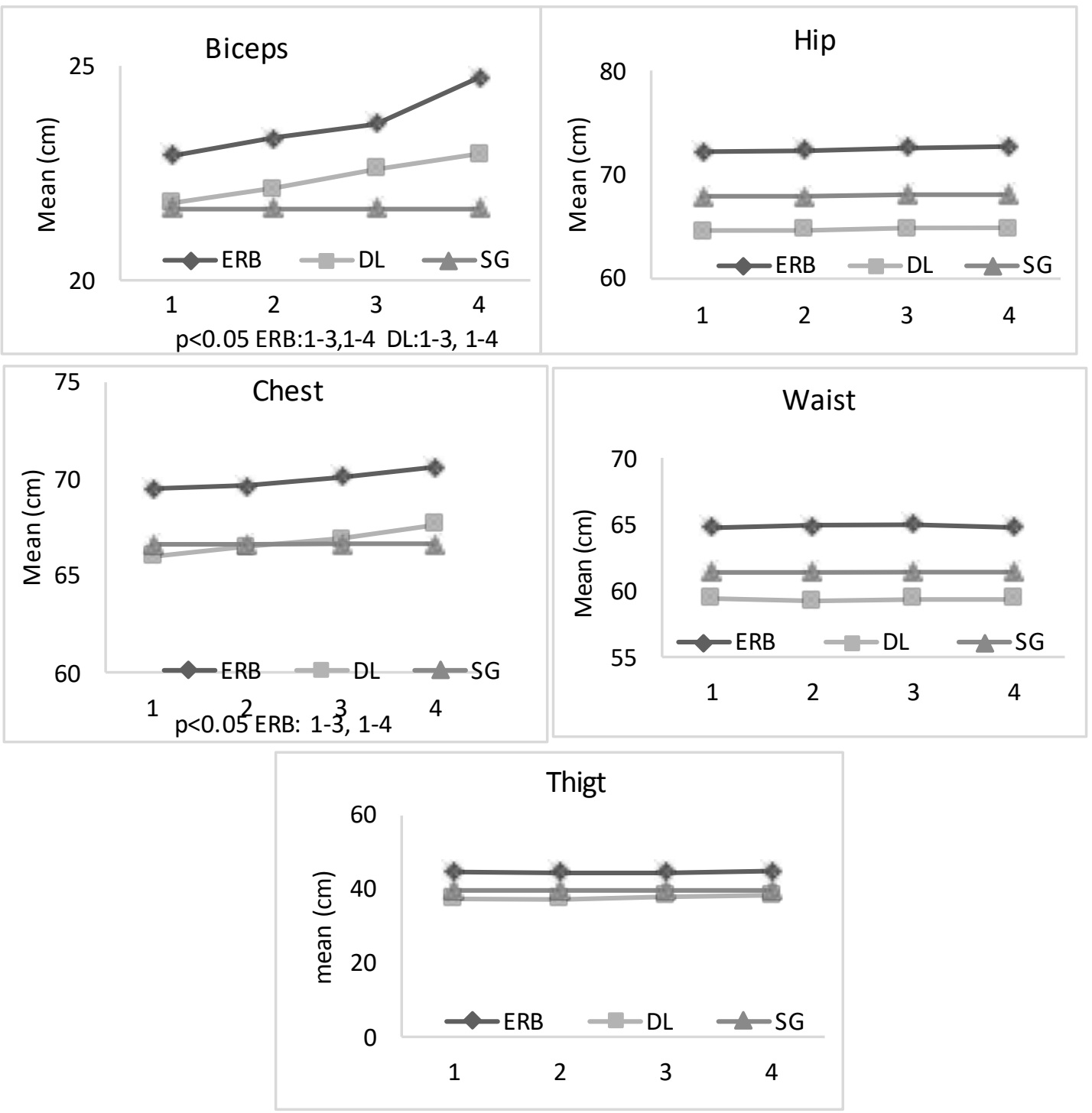

Figure 1. The circumference variables according to the groups. 

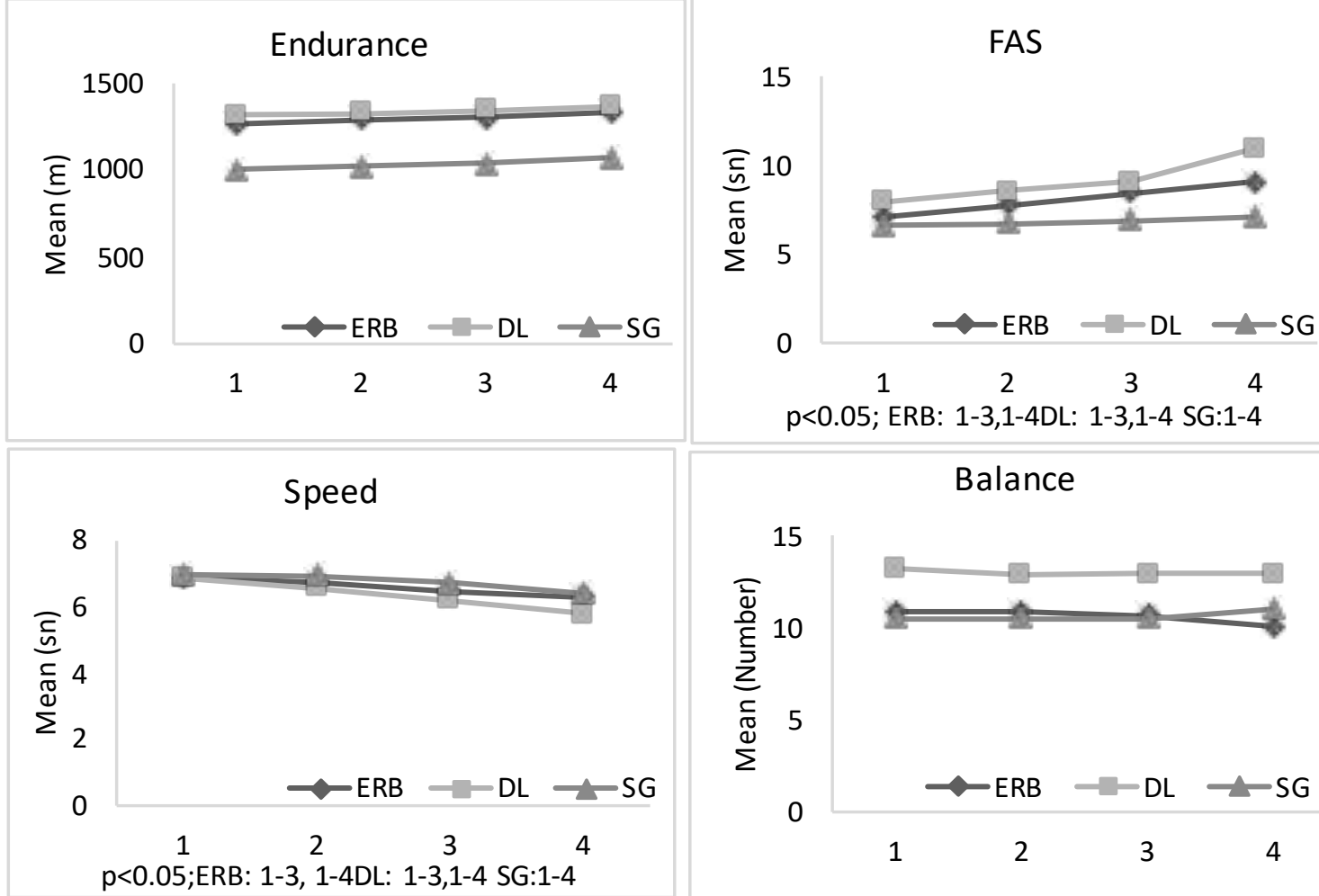

Balance
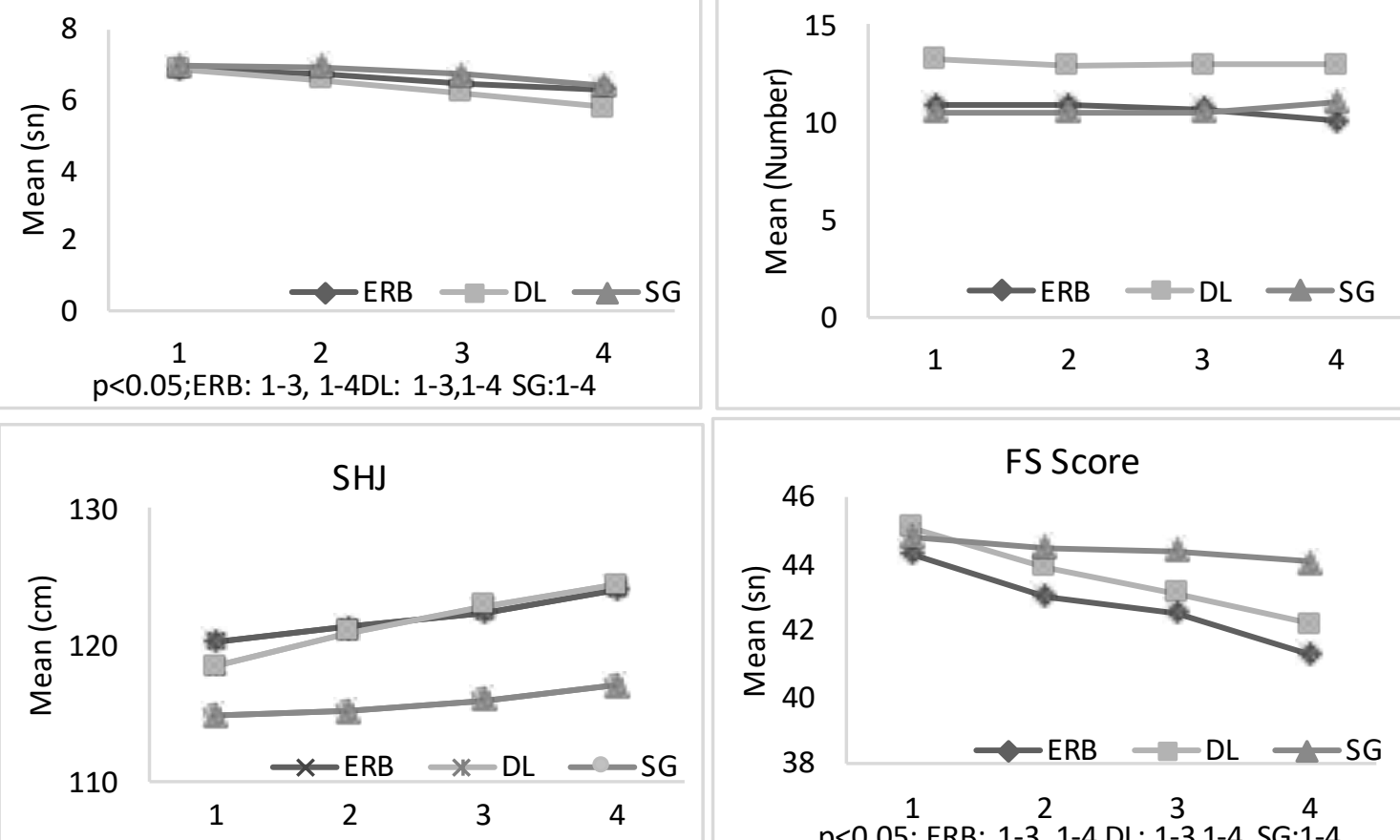

p<0.05; ERB: 1-3, 1-4 DL: 1-3,1-4 SG:1-4
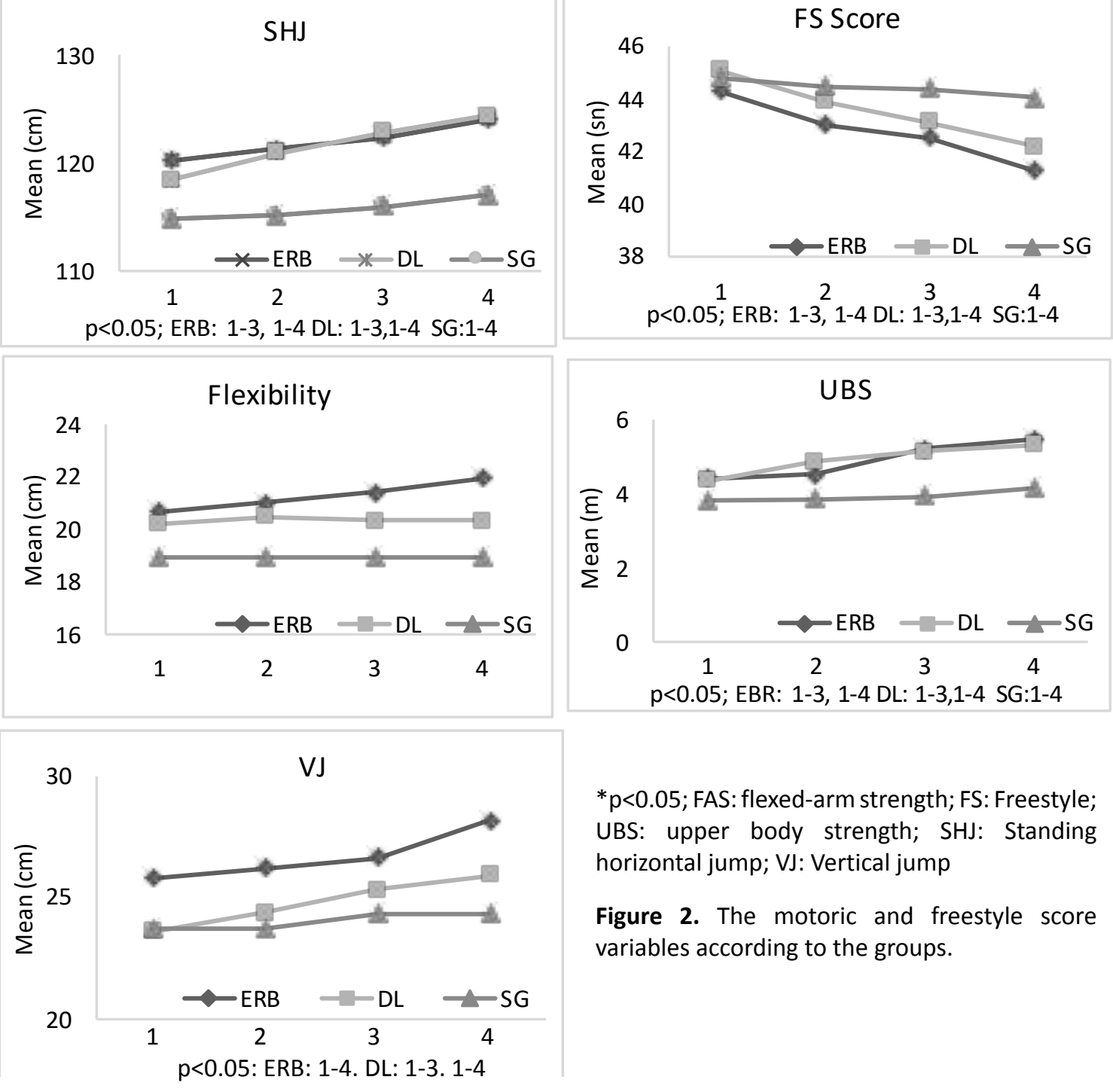

${ }^{*} p<0.05$; FAS: flexed-arm strength; FS: Freestyle; UBS: upper body strength; SHJ: Standing horizontal jump; VJ: Vertical jump

Figure 2. The motoric and freestyle score variables according to the groups. 
Table 3. The performance variables as based on the assessment times and total change difference.

\begin{tabular}{|c|c|c|c|c|c|c|}
\hline \multirow{2}{*}{ Variables } & TM & ERB $^{a}$ & Dry-land ${ }^{b}$ & Swimming ${ }^{c}$ & $\mathbf{p}$ & Post-Hoc p \\
\hline & & $M \pm S D$ (median) & $M \pm S D$ (median) & \multicolumn{3}{|l|}{$\mathrm{M} \pm \mathrm{SD}$ (median) } \\
\hline \multirow{4}{*}{ VJ $(\mathrm{cm})$} & 1 & $25.80 \pm 4.0(27)$ & $23.60 \pm 2.9(23)$ & $23.73 \pm 4.6(24)$ & - & - \\
\hline & 2 & $26.20 \pm 4.3(27)$ & $24.39 \pm 2.7(23.7)$ & $23.73 \pm 4.6(24)$ & 0.001 & $0.001(b-c)$ \\
\hline & 3 & $26.66 \pm 4.5(27)$ & $25.34 \pm 2.5(25)$ & $24.33 \pm 3.9(24)$ & 0.010 & $0.007(b-c)$ \\
\hline & 4 & $28.2 \pm 4.4(29)$ & $25.90 \pm 2.5(25.8)$ & $24.33 \pm 3.9(24)$ & 0.001 & $\begin{array}{l}0.005(a-c) \\
0.004(b-c)\end{array}$ \\
\hline TCD & & $9.3 \%$ & $9.4 \%$ & $2.5 \%$ & 0.001 & $a-c ; b-c$ \\
\hline \multirow{6}{*}{ FAS (s) } & 1 & $7.08 \pm 3.6(6.4)$ & $7.92 \pm 2.6(7.8)$ & $6.62 \pm 2.0(6.4)$ & - & - \\
\hline & \multirow[t]{2}{*}{2} & \multirow{2}{*}{$7.75 \pm 3.5(7.1)$} & \multirow{2}{*}{$8.51 \pm 2.5(8.1)$} & \multirow{2}{*}{$6.68 \pm 2.0(6.5)$} & \multirow{2}{*}{0.001} & $0.006(a-c)$ \\
\hline & & & & & & $\begin{array}{l}0.003(b-c) \\
0.015(a-c)\end{array}$ \\
\hline & 3 & $8.46 \pm 2.9(8.1)$ & $9.02 \pm 2.4(8.5)$ & $6.91 \pm 2.1(6.7)$ & 0.002 & $0.004(b-c)$ \\
\hline & \multirow{2}{*}{4} & \multirow{2}{*}{$9.08 \pm 2.9(9.1)$} & \multirow{2}{*}{$10.95 \pm 2.7(10.7)$} & $708+21(72)$ & $<\cap 001$ & $0.003(a-c)$ \\
\hline & & & & $1.00 \pm 2.1(1.2)$ & $<0.001$ & $0.001(b-c)$ \\
\hline TCD & & $28.2 \%$ & $38.3 \%$ & $6.9 \%$ & 0.001 & $a-c ; b-c$ \\
\hline & 1 & $6.88 \pm 0.5(6.1)$ & $6.87 \pm 05(6.7)$ & $6.98 \pm 05$ (6.9) & - & - \\
\hline & 2 & $6.75 \pm 0.4(6.1)$ & $6.58 \pm 04(6.4)$ & $6.94 \pm 05(6.9)$ & 0.083 & - \\
\hline Speed (s) & 3 & $6.49 \pm 0.5(5.8)$ & $6.21 \pm 03(5.9)$ & $6.72 \pm 05(6.6)$ & 0.017 & $0.014(b-c)$ \\
\hline & 4 & $6.29+0.5(5.6)$ & $5.81+03(5.7)$ & $641+04(62)$ & م008 & $0.027(a-b)$ \\
\hline & & & (5.) & $0.41 \pm 04(0.2)$ & 0.000 & $0.016(b-c)$ \\
\hline TCD & & $8.6 \%$ & $15.4 \%$ & $8.1 \%$ & 0.008 & $a-b ; b-c$ \\
\hline & 1 & $4.40 \pm 1.0(4.5)$ & $4.34 \pm 0.6(4.5)$ & $3.82 \pm 1.1(4.1)$ & - & - \\
\hline & 2 & $4.52 \pm 0.9(4.5)$ & & & & $0.020(a-c)$ \\
\hline & 2 & $4.5 \angle \pm 0.9(4.5)$ & $4.88 \pm 0.5(4.9)$ & $3.8 / \pm 1.0(4.1)$ & 0.001 & $0.001(b-c)$ \\
\hline UBS (m) & & & & & & 0.002 (a-c) \\
\hline & 3 & $5.22 \pm 0.9(5.2)$ & $5.14 \pm 0.5(5.2)$ & $3.93 \pm 0.9(4.2)$ & $<0.00$ & $0.001(b-c)$ \\
\hline & & & & & & 0.002 (a-c) \\
\hline & 4 & $5.47 \pm 0.8(5.7)$ & $5.31 \pm 6.6(5.2)$ & $4.15 \pm 0.7(4.2)$ & 0.001 & $0.009(b-c)$ \\
\hline TCD & & $24.3 \%$ & $22.4 \%$ & $8.6 \%$ & 0.001 & $a-c ; b-c$ \\
\hline & 1 & $120.20 \pm 9.5(121)$ & $118.46 \pm 8.5(120)$ & $114.83 \pm 8.6(118)$ & - & - \\
\hline cis laml & 2 & $121.26 \pm 9.3(124)$ & $120.98 \pm 7.5(123.7)$ & $115.06 \pm 8.8(119)$ & 0.075 & - \\
\hline $\mathrm{SHJ}(\mathrm{cm})$ & 3 & $122.38 \pm 7.9(125)$ & $122.82 \pm 7.6(124.5)$ & $115.93 \pm 7.6(120)$ & 0.075 & - \\
\hline & 4 & $124.00 \pm 7.7(125)$ & $124.40 \pm 7.3(125)$ & $117.06 \pm 6.4(120)$ & 0.088 & - \\
\hline TCD & & $3.2 \%$ & $5.0 \%$ & $1.9 \%$ & & NS \\
\hline & 1 & $20.66 \pm 6.1(21)$ & $20.20 \pm 4.4(21)$ & $18.93 \pm 4.3(20)$ & - & - \\
\hline Flexibility & 2 & $21.00 \pm 6.0(21)$ & $20.46 \pm 3.8(21)$ & $18.93 \pm 4.3(20)$ & 0.173 & - \\
\hline$(\mathrm{cm})$ & 3 & $21.40 \pm 5.4(21)$ & $20.33 \pm 3.8(20)$ & $18.93 \pm 4.3(20)$ & 0.079 & - \\
\hline & 4 & $21.93 \pm 5.3(21)$ & $20.33 \pm 3.8(20)$ & $18.93 \pm 4.3(20)$ & 0.068 & - \\
\hline TCD & & $6.1 \%$ & $0.6 \%$ & $0 \%$ & & NS \\
\hline & 1 & $1266 \pm 271(1300)$ & $1320 \pm 221(1300)$ & $1006 \pm 116(1000)$ & - & - \\
\hline Endurance & 2 & $1293 \pm 234(1300)$ & $1326 \pm 212(1300)$ & $1020 \pm 108(1000)$ & 0.541 & - \\
\hline$(\min )$ & 3 & $1306 \pm 221(1300)$ & $1346 \pm 195(1300)$ & $1040 \pm 91(1100)$ & 0.718 & - \\
\hline & 4 & $1333 \pm 191(1300)$ & $1366 \pm 183(1300)$ & $1073 \pm 103(1100)$ & 0.670 & - \\
\hline TCD & & $5.3 \%$ & $3.5 \%$ & $6.6 \%$ & & \\
\hline & 1 & $10.86 \pm 5.9(10)$ & $13.20 \pm 4.7(14)$ & $10.46 \pm 4.7(10)$ & - & - \\
\hline Dalama & 2 & $10.86 \pm 4.7(10)$ & $12.86 \pm 4.1(14)$ & $10.46 \pm 4.7(10)$ & 0.563 & - \\
\hline Balance & 3 & $10.66 \pm 3.8(10)$ & $12.93 \pm 3.7(14)$ & $10.46 \pm 4.7(10)$ & 0.589 & - \\
\hline & 4 & $10.06 \pm 3.5(10)$ & $12.93 \pm 3.7(14)$ & $11.00 \pm 4.7(10)$ & 0.159 & - \\
\hline TCD & & $7.4 \%$ & $2.0 \%$ & $5.0 \%$ & & NS \\
\hline
\end{tabular}

p<0.05; Abbrevations: cm: centimeter; $\mathbf{m}$ : meter; min: minute; NS: non-significant; s: second; TCD: Between $1^{\text {st }}$ and $4^{\text {th }}$ measurement the total change difference. TM: times of measurement [(the beginning of the study (1), and after four (2), eight (3) and twelve- week of the training session (4)]. 
Table 4. The FS performance as based on the assessment times and total change difference.

\begin{tabular}{|c|c|c|c|c|c|c|c|}
\hline \multirow[b]{2}{*}{ Variable } & & \multirow{2}{*}{$\begin{array}{l}\mathrm{ERB}^{\mathrm{a}} \\
\mathrm{M} \pm \mathrm{SD} \\
\text { (median) }\end{array}$} & \multirow{2}{*}{$\begin{array}{l}\text { Dry-land } \\
\mathrm{M} \pm \mathrm{SD} \\
\text { (median) }\end{array}$} & \multicolumn{2}{|l|}{ Swimming ${ }^{c}$} & \multicolumn{2}{|l|}{ Post-Hoc } \\
\hline & & & & $\begin{array}{l}M \pm S D \\
\text { (median) }\end{array}$ & $\mathbf{p}$ & & \\
\hline \multirow{12}{*}{$\begin{array}{l}\text { FS Score } \\
\text { (s) }\end{array}$} & 1 & $44.27 \pm 1.8(44.1)$ & $45.06 \pm 2.2(45.4)$ & $44.79 \pm 1.9(45.2)$ & - & - & \multirow{12}{*}{$\begin{array}{l}(a-c) \\
(b-c)\end{array}$} \\
\hline & 2 & $43.02 \pm 1.8(42.8)$ & $43.89 \pm 1.8(44.2)$ & $44.50 \pm 1.7(45.2)$ & 0.002 & 0.021 & \\
\hline & & & & & & 021 & \\
\hline & CD (1-2) & $2.82 \%$ & $2.59 \%$ & $0.64 \%$ & 0.002 & 0002 & \\
\hline & & & & & & 0.012 & \\
\hline & 3 & $42.55 \pm 1.7(41.2)$ & $43.12 \pm 1.7(43.5)$ & $44.39 \pm 1.8(45.1)$ & $<0.001$ & 0.001 & \\
\hline & & & & & & 0.012 & \\
\hline & CD (1-3) & $3.88 \%$ & $4.30 \%$ & $0.89 \%$ & 0.000 & 0.001 & \\
\hline & & & & & & 0.001 & \\
\hline & 4 & $41.27 \pm 1.4(40.2)$ & $42.21 \pm 1.6(42.8)$ & $44.07 \pm 1.7(44.8)$ & $<0.001$ & 0.001 & \\
\hline & TCD (1-4) & $6.77 \%$ & $6.32 \%$ & $1.6 \%$ & 0.000 & 0.000 & \\
\hline & & & & & & 0.000 & \\
\hline
\end{tabular}

$\mathbf{p}<0.05$; CD: Between measurement change differences; s: second; FS: Freestyle; TCD: Between $1^{\text {st }}$ and $4^{\text {th }}$ measurement the total change difference.

DL and $\mathrm{SG}(\mathrm{p}<0.001)$. Besides, there was a significant difference in the fourth measurement time between the ERB and DL $(p<0.001)$. In TCD, there was a significant change both between the ERB and DL training group, and besides, the change difference was significantly in DL training group comparing to the swimming group $(p<0.05)$. In UBS performance change, there was a significant difference between ERB and DL, and also DL and swimming in second measurement. In the third and fourth measurements, there was a significant difference in both ERB and DL versus $S G(p<0.001)$. In TCD, there was no significant change between the ERB and DL training group, but the change difference was significantly in ERB and DL training group comparing to the $\mathrm{SG}(\mathrm{p}<0.05)$. In other variables, there was no significant difference among the groups in any measurement times.

In FS performance change, there was a significant difference in both of ERB and DL training groups versus SG in all measurements except the first $(p<0.001)$. In TCD, there was no significant change between the ERB and DL training group, but the change difference was significantly in ERB and DL training group comparing to the swimming group $(\mathrm{p}<0.05)$.

\section{Discussion}

The first purpose of the study is to compare the effects of 12- weeks of the dry-land training (ERB and DL) that are named as experimental and in-water training, named as swimming (SG). The main findings of the study showed that experimental groups had statistically significant increase in both VJ and SHJ performance conditions compared to the SG (Figure 2). Besides, it showed that all groups had statistically significant increases in speed, UBS, FAS, but the experimental groups accessed the improvements at the end of week 8 of the study, while the SG had the improvements at week 12 of the study (Figure 2). However, no statistically significant improvements were observed in balance, endurance, and flexibility for all groups. But it was observed that flexibility was improved more relatively in ERB group compare the other groups (Figure 2, Table 3). Paralleled to these finding, biceps circumference statistically increased in experimental groups, while just chest circumference increased in ERB group (Figure 1). Improvement in FS score occurred after 12 weeks of ERB and DL training groups with the rate of $6.77 \%$ and $6.32 \%$ respectively compared to SG $1.6 \%$. Besides, it was seen statistically significant improvement for both of experiment group, but in ERB group the improvement was relatively more compared to DL group (Figure 2; Table 4). Some of the studies that used different training protocols in-water, Girold et al. [25] reported that combining swimming and dry-land strength or swimming and resisted and assisted sprint were more efficient than only the swimming program in increasing sprint performance in 50-meter front crawl swimming. The researchers observed that there were no differences between dry-land strength training and in-water resistedand assisted-sprint training methods. Colado et al. [26], found that the training using aquatic devices in-water was as effective as training using ERB or weight machines to improve the physical capacity and body composition of postmenopausal women. In other studies, Breed and Young [27] stated that resistance training modes with free weight or machine improved leg power and jumping ability. Besides, the findings showed that improved jumping ability increased the vertical force components of on the grab, track and swing starts in swimming. According to the other study, Morais et al. [28] stated 
that the swimmers with higher body dimensions achieved better performances in the squat jump, countermovement jump, throwing velocity tests. Amaro et al. [5], reported that 6 weeks of complementary dry-land training led to improvements on strength and explosive, after a 4-week adaptation period for the swimmers. The explosive actions such as starts and turns are highly important on swimming performance that related relative contribution of anaerobic pathways to power production. Therefore, the improving muscle strength seems to be crucial for enhancing competitive swimming performance, and will lead to an increased ability to produce propulsive force in the water. In particular, the upper-body strength, that leads to producing most of the propulsive forces and swimming velocity, is produced as a necessity [29]. The studies supporting this hypothesis, Muniz-Pardos et al. [30] reported that the strength of the upper limbs provides approximately $75 \%$ of the energy required for an efficient propulsive force during front crawl, while the lower body strength contributed to the propulsive forces in low to moderate ways, but it had a huge effect during the start and turn phases. Pérez-Olea et al. [31] reported that upperlimb explosive strength was significantly correlated with the swimming performance, but there was no significant relationship between measures of lower-limb strength values and swimming performance. Sammoud et al. [32], reported that plyometric jump training together with the swimming training was more effective than just regular swimming training in improving jump and swimming performances. In another study, Andersen et al. [33], implemented full-body elastic resistance band program, before the regular handball training sessions. The researches [33], reported that the training improved explosive lower-limb performance, jump height, power output, and average velocity in the squat more than compared to the handball training alone. The literature and our findings confirm that engaged in the ERB or DL training program with a focus on gaining strength and power in particular upper body strength, has seemed like an important training part that should be added to the common swimming training schedule.

The second aim of the study was to assess the effectiveness of ERB and DL resistance trainings used and which one was (more) effective in enhancing the performance. The main findings of both training models showed similar level of improvements in performance components. But, DL training led to more improvements relatively in both FAS (38.3\%) and speed (15.4\%) performance in comparison with the ERB training regimen (FAS: $28.2 \%$; speed: $8.6 \%$ ) at the end of the study. Besides, a statistically significant difference was found in speed performance between the ERB and DL training. However, no statistically significant improvements were seen in flexibility, balance and aerobic endurance during the study among both of training models (Table 3), but it was observed that flexibility was improved more relatively in ERB group $(6.1 \%)$ compare the DL group $(0.6 \%)$ (Figure 2, Table 3$)$. A study finding that focused on what type of training was executed by swimmers according to their age groups reported that the age groups of $\leq 10$ and $11-14$ (years) spent $28 \%-40 \%$ on DL training, whereas the collegiate and master's groups spent $21 \%$ and $15 \%$, respectively [34]. They stated that the $\leq 10$ (years) age group spent less than 2 hours per week on dry-land training. Besides, Krabak et al. [34], stated that modes of training in younger swimmers ( $\leq 10$ and $11-14$ years) consisted of percent values with EBR: $7 \%$, body weight: 40\%, medicine ball: $4 \%$; ERB: $11 \%$, body weight: $10 \%$, medicine ball: $10 \%$ respectively. The literature focusing on the effects of the different training methods, Özsu [35], found that ERB exercises increased hand-grip strength, but did not improve flexibility and agility performance. Therefore, Özsu [35], reported that 6-week elastic resistance band exercises could be helpful to increase the muscular fitness level of children in 8-9 age groups. Similarly, Şahin et al. [7] stated that elastic band training led to more improvements in static squat and vertical jump performance. They reported that ERB compared to the body weight was only developed better in pre-adolescents. Janusevicius et al. [17] found that resistance training with elastic band at high movement velocity improved sprint performance. Janusevicius et al. [17] suggest that also suggested that elastic band training at high movement velocity would increase the overall hamstring muscle power output more, compared to the heavy resistance training. In our study, DL training showed a significant improvement in speed performance compared to the ERB training (rate of $15.4 \%$ and $8.6 \%$ respectively) (Table 3 ). In other studies, Joy et al. found that variable resistance training with the use of elastic bands resulted in greater changes in the rate of power development than the SG. Moreover, the training led to greater increases in squat, bench press, and all jumping measurements. Batalha et al. [36] reported that the strength training with the elastic band may lead to help swimmers reduce the risk of injury by increasing shoulder rotator strength and preventing any shoulder muscle imbalances. Similarly, Mascarin et al. [37] showed that strength training using elastic bands before regular handball training was effective to improve muscle power in shoulder internal and external-rotator muscle performance. They reported that the training with elastic bands presented higher values in ball speed with standing and jumping throws.

Based on the literature and our results, the findings support the use of the ERB / DL according to the training goals. Besides, a variety of training regimens versus only one way should be employed to augment strength and power. In fact, all the findings in studies in the literature and also ours support that any type of resistance training, not only just swimming, could be effective. It helps developing performance components to changeable rates based on the training aims. The findings suggest that each of the performance components should be included within the private training regimens that effected it directly.

\section{Conclusions}

According to the findings, each of the training modalities may lead to focus on different performance 
components. DL training provided more improvements the variables, including VJ, UBS, speed, FAS, SHJ compared to both of ERB and SG. ERB training also occurred more improvement in flexibility and balance according to the other groups, but swimming training was only effective on endurance performance. Besides, the findings showed also that ERB training provided relatively more improvement on swimming performance than the other groups. Based on the finding, it may be said that as the reason for the improvement of swimming performance, it caused elastic band movements that used in ERB training stem from the similar to the techniques used in swimming.

\section{Conflicts of interest}

The authors declare no conflict of interest.

\section{References}

1. De Assis Correia R, Feitosa WG, Figueiredo P, Papoti M, De Souza Castro FA. The 400-m front crawl test: Energetic and $3 \mathrm{~d}$ kinematical analyses. International Journal of Sports Medicine. 2020; 41(1): 21-26. https://doi.org/10.1055/a-1023-4280

2. Yapıcı Öksüzoğlu A. The effects of theraband training on respiratory parameters, upper extremity muscle strength and swimming performance. Pedagogy of Physical Culture and Sports, 2020; 24(6), 316-322. https://doi.org/10.15561/26649837.2020.0607

3. Ribeiro J, Figueiredo P, Sousa A, Monteiro J, Pelarigo J, VilasBoas JP, et al. $\mathrm{VO}_{2}$ kinetics and metabolic contributions during full and upper body extreme swimming intensity. European Journal of Applied Physiology. 2015; 115(5): 1117-1124. https://doi.org/10.1007/s00421-014-3093-5

4. Silva AF, Figueiredo P, Ribeiro J, Alves F, Vilas-Boas JP, Seifert L, et al. Integrated analysis of young swimmers' sprint performance. Motor Ccontrol. 2019; 23(3): 354-364. https://doi.org/10.1123/mc.2018-0014

5. Amaro NM, Marinho DA, Marques MC, Batalha NP, Morouço PG. Effects of dry-land strength and conditioning programs in age group swimmers. Journal of Strength and Conditioning Research. 2017; 31(9): 2447-2454. https://doi.org/10.1519/JSC.0000000000001709

6. Skucas K, Pokvytyte V. Combined strength exercises on dry land and in the water to improve swimming parameters of athletes with paraplegia. The Journal of Sports Medicine and Physical Fitness. 2018; 58(3): 197-203. https://doi.org/10.23736/S0022-4707.16.06702-5

7. Şahin G, Aslan M, Demir E. Short-term effect of back squat with an elastic band on the squat and vertical jump performance in trained children. Journal of Physical Education and Sport. 2016; 16(1): 97-101. https://doi.org/10.7752/jpes.2016.01016

8. Morouço P, Keskinen KL, Vilas-Boas JP, Fernandes RJ. Relationship between tethered forces and the four swimming techniques performance. Journal of Applied Biomechanics. 2011; 27(2): 161-169. https://doi.org/10.1123/jab.27.2.161

9. Strzała M, Tyka A. Physical Endurance, Somatic indices and swimming technique parameters as determinants of front crawl swimming speed at short distances in young swimmers. Medicina Sportiva. 2009; 13(2): 99-107.

10. Tanaka H, Costill DL, Thomas R, Fink, WJ, Widrick, JJ. Dryland resistance training for competitive swimming. Medicine and Science in Sports and Exercise. 1993; 25(8): 952-959.

11. Myers AM, Beam NW, Fakhoury JD. Resistance training for children and adolescents. Translational Pediatrics. 2017; 6(3): 137-143. https://doi.org/ 10.21037/tp.2017.04.01

12.Cuenca-Fernández F, Gay A, Ruiz-Navarro JJ, Arellano R. The effect of different loads on semi- tethered swimming and its relationship with dryland performance variables. International Journal of Performance Analysis in Sport. 2020; 20(1): 90-106. https://doi.org/10.1080/24748668.2020.1714413

13.Pekünlü E. Çocuklar ve gençlerde direnç antrenmanı. Türkiye Klinikleri Journal of Sports Sciences. 2019; 11(1): 29-40. https://doi.org/10.5336/sportsci.2018-63406

14.Aloui G, Hammami M, Fathloun M, Hermassi S, Gaamouri $\mathrm{N}$, Shephard RJ, et al. Effects of an 8-week in-season elastic band training program on explosive muscle performance, change of direction, and repeated changes of direction in the lower limbs of junior male handball players. Journal of Strength and Conditioning Research. 2019;33(7): 1804-1815. https://doi.org/10.1519/JSC.0000000000002786

15.Melchiorri G, Rainoldi A. Muscle fatigue induced by two different resistances: Elastic tubing versus weight machines. Journal of electromyography and kinesiology: Official journal of the International Society of Electrophysiological Kinesiology. 2011; 21(6): 954-959. https://doi.org/10.1016/j.jelekin.2011.07.015

16.Andersen V, Fimland MS, Kolnes MK, Saeterbakken AH. Elastic bands in combination with free weights in strength training: neuromuscular effects. Journal of Strength and Conditioning Research. 2015; 29(10): 2932-2940. https://doi.org/10.1519/JSC.0000000000000950

17.Janusevicius D, Snieckus A, Skurvydas A, Silinskas V, Trinkunas E, Cadefau JA, et al. Effects of high velocity elastic band versus heavy resistance training on hamstring strength, activation, and sprint running performance. Journal of Sports Science \& Medicine. 2017; 16(2): 239-246.

18.Joy JM, Lowery RP, Oliveira de Souza E, Wilson JM. Elastic Bands as a Component of Periodized Resistance Training. Journal of Strength and Conditioning Research. 2016; 30(8): 2100-2106. https://doi.org/10.1519/JSC.0b013e3182986bef

19.Chang TF, Liou TH, Chen CH, Huang YC, Chang KH. Effects of elastic-band exercise on lower-extremity function among female patients with osteoarthritis of the knee. Disability and Rehabilitation. 2012; 34(20): 1727-1735. https://doi.org/10.3109/09638288.2012.660598

20.Martins WR, de Oliveira RJ, Carvalho RS, de Oliveira Damasceno V, da Silva VZ, Silva MS. Elastic resistance training to increase muscle strength in elderly: a systematic review with meta-analysis. Archives of Gerontology and Geriatrics. 2013; 57(1): 8-15. https://doi.org/10.1016/j.archger.2013.03.002

21.Ercan O. Physical development of the adolescent. İ.Ü. Cerrahpaşa Faculty of Medicine Continuing Medical Education Activities. Adolescent Health II. 2008; 63: 13-18. (In Turkish).

22.Sands WA, Wurth JJ, Hewit JK. Basics of strength and conditioning manual. The National Strength and Conditioning Association's (NSCA); 2012. 
23.Miller DK. Measurement by the physical educator why and how. St. Wilmington: Mc Graw Hill; 2006.

24.Adams MG, Beam CW. Exercise physiology laboratory manual. (7 th ed) St. Wilmington: Mc Graw Hill; 2008.

25. Girold S, Maurin D, Dugué B, Chatard JC, Millet G. Effects of dry-land vs. resisted- and assisted-sprint exercises on swimming sprint performances. Journal of Strength and Conditioning Research. 2007; 21(2): 599-605. https://doi.org/10.1519/R-19695.1

26.Colado JC, Garcia-Masso X, Rogers ME, Tella V, Benavent J, Dantas EH. Effects of aquatic and dry land resistance training devices on body composition and physical capacity in postmenopausal women. Journal of Human Kinetics. 2012; 32: 185-195. https://doi.org/10.2478/v10078-012-0035-3

27.Breed RV, Young WB. The effect of a resistance training programme on the grab, track and swing starts in swimming. Journal of Sports Sciences. 2003; 21(3): 213-220. https://doi.org/10.1080/0264041031000071047

28. Morais JE, Silva AJ, Marinho DA, Marques MC, Barbos, TM. Effect of a specific concurrent water and dry-land training over a season in young swimmers' performance. International Journal of Performance Analysis in Sport. 2016; 16(3): 760-775.

29.Schumann M, Rønnestad BR. Concurrent aerobic and strength training: Scientific basics and practical applications. Springer; 2019.

30.Muniz-Pardos B, Gomez-Bruton A, Matute-Llorente A, Gonzalez-Aguero A, Gomez-Cabello A, GonzaloSkok O, et al. Swim-Specific Resistance Training: A Systematic Review. Journal of Strength and Conditioning Research. 2019; 33(10): 2875-2881. https://doi.org/10.1519/JSC.0000000000003256

31.Pérez-Olea JI, Valenzuela PL, Aponte C, Izquierdo M. Relationship between dryland strength and swimming performance: pull-up mechanics as a predictor of swimming speed. Journal of Strength and Conditioning Research. 2018; 32(6): 1637-1642. https://doi.org/10.1519/JSC.0000000000002037

32.Sammoud S, Negra Y, Chaabene H, Bouguezzi R, Moran $\mathrm{J}$, Granacher U. The effects of plyometric jump training on jumping and swimming performances in prepubertal male swimmers. Journal of Sports Science \& Medicine. 2019; 18(4): 805-811.

33.Andersen V, Fimland MS, Cumming KT, Vraalsen $\varnothing$, Saeterbakken AH. Explosive resistance training using elastic bands in young female team handball players. Sports Medicine International Open. 2018; 2(6): E171-E178. https://doi.org/10.1055/a-0755-7398

34.Krabak, BJ, Hancock KJ, Drak S. Comparison of dry-land trainingprogramsbetweenagegroupsofswimmers. TheJournal of Injury, Function, and Rehabilitation. 2013; 5(4): 303-309. https://doi.org/10.1016/j.pmrj.2012.11.003

35.Özsu I. Effects of 6-week resistance elastic band exercise on functional performances of 8-9 year-old children. Journal of Education and Training Studies. 2018; 6: 23-28.

36.Batalha N, Raimundo A, Tomas-Carus P, Paulo J, Simão R, Silva AJ. Does a land-based compensatory strength-training programme influences the rotator cuff balance of young competitive swimmers?. European Journal of Sport Science. 2015; 15(8): 764-772. https://doi.org/10.1080/17461391.2015.1051132

37.Mascarin NC, de Lira C, Vancini RL, de Castro Pochini A, da Silva AC, Dos Santos Andrade M. Strength training using elastic bands: improvement of muscle power and throwing performance in young female handball players. Journal of Sport Rehabilitation. 2017; 26(3): 245-252. https://doi.org/10.1123/jsr.2015-0153

\section{Information about the authors:}

İsmet Alagöz; https://orcid.org/0000-0002-2538-3699; alagoz.ismet@gmail.com; Ministry of Youth and Sports; Çorum, Turkey.

Sema Can; (Corresponding author); https://orcid.org/0000-0003-1931-2226; semacan@hitit.edu.tr; Faculty of Sport Sciences, Department of Coaching Training, Hitit University; Çorum, Turkey.

Erkan Demirkan; https://orcid.org/0000-0002-6243-8062; erkandemirkan@hitit.edu.tr:Faculty of Sport Sciences, Department of Coaching Training, Hitit University; Çorum, Turkey.

Tuğrul Özkadı; https://orcid.org/0000-0001-9336-6957; tugrulozkadi@hitit.edu.tr; Department of Sport Management, Hitit University; Çorum, Turkey.

Emre Demir; https://orcid.org/0000-0002-3834-3864; emredemir@hitit.edu.tr; Faculty of Medicine, Deparment of Biostatistics, Hitit University; Çorum, Turkey.

Cite this article as:

Alagöz İ, Can S, Demirkan E, Özkadı T, Demir E. Effect of different training models on motoric and swimming performance in prepubescent swimmers. Pedagogy of Physical Culture and Sports, 2021;25(5):286-295. https://doi.org/10.15561/26649837.2021.0503

This is an Open Access article distributed under the terms of the Creative Commons Attribution License, which permits unrestricted use, distribution, and reproduction in any medium, provided the original work is properly cited (http://creativecommons.org/licenses/by/4.0/deed.en).

Received: 15.04.2021

Accepted: 18.06.2021; Published: 30.10.2021 